\title{
Online cognitive behavioral therapy for insomnia (CBT-I) for the treatment of insomnia among individuals with alcohol use disorder: study protocol for a randomized controlled trial
}

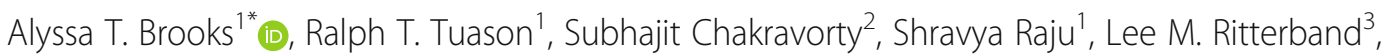
Frances P. Thorndike ${ }^{4}$ and Gwenyth R. Wallen ${ }^{1}$

\begin{abstract}
Alcohol use disorder (AUD) is characterized by problematic drinking that becomes severe. Individuals with AUD often experience insomnia and other sleep disturbances at various phases of recovery. Cognitive behavioral therapy for insomnia (CBT-I) is an efficacious non-pharmacological treatment for insomnia and is recommended as a first-line treatment for adults with chronic insomnia. Internet-based CBT-I could play a key role in the dissemination of this behavioral sleep intervention, given the paucity of trained clinicians able to provide CBT-I in person and other logistical/cost concerns. SHUTi (Sleep Healthy Using The Internet) is the most tested and empirically-sound Internet intervention for insomnia. Despite the promise of Internet-based CBT-I interventions, to date, no randomized controlled trials (RCTs) exist examining the feasibility/efficacy of an Internet-based CBT-I program among treatment-seeking individuals recovering from AUD. This is a two-phase RCT assessing feasibility/acceptability and efficacy of the SHUTi program among individuals with AUD in recovery with insomnia. Phase I will focus on assessing the feasibility and acceptability of program delivery and data collection $(n=10)$. Phase II will be an RCT powered to examine preliminary intervention efficacy ( $n=30$ per group). Participants for this study must meet criteria for "moderate to severe" insomnia. Individuals randomized to the intervention group will receive the SHUTi intervention (initiated while inpatient and completed while outpatient), and individuals randomized to the control group will receive an educational web-based program. The goals of the study are as follows: (1) assess the feasibility and acceptability of Internet-based CBT-I among individuals with AUD in recovery with insomnia (phase I), (2) compare the preliminary efficacy of CBT-I versus control group with respect to primary and secondary outcome variables (phase II), and (3) explore specific domains associated with improved outcomes, e.g., demographic, psychiatric, and drinking-related factors (phase II). Primary outcome measures include changes in insomnia severity over time and changes in actigraphy-recorded sleep efficiency over time.
\end{abstract}

Trial registration: NCT\#03493958; registered 1 June 2018.

Keywords: Alcohol use disorder, Insomnia, Cognitive behavioral therapy for insomnia, Sleep disturbance, Internet intervention

\footnotetext{
* Correspondence: todaroad@mail.nih.gov

${ }^{1}$ National Institutes of Health Clinical Center, Bethesda, USA

Full list of author information is available at the end of the article
}

(c) The Author(s). 2018 Open Access This article is distributed under the terms of the Creative Commons Attribution 4.0 International License (http://creativecommons.org/licenses/by/4.0/), which permits unrestricted use, distribution, and reproduction in any medium, provided you give appropriate credit to the original author(s) and the source, provide a link to the Creative Commons license, and indicate if changes were made. The Creative Commons Public Domain Dedication waiver (http://creativecommons.org/publicdomain/zero/1.0/) applies to the data made available in this article, unless otherwise stated. 


\section{Background}

\section{Alcohol and sleep disturbances}

Alcohol use disorder (AUD) is characterized by problematic drinking that becomes severe [1]. To be diagnosed with "severe" AUD, individuals must meet six or more of the 11 criteria outlined in the Diagnostic and Statistical Manual (current edition: DSM-5). This was referred to as "alcohol dependence" in previous versions of the DSM; thus, some researchers and clinicians still use the term "dependence" and research conducted prior to the release of the DSM-5 uses the term "dependence." Alcohol dependence is associated with insomnia and a myriad of other sleep-related disorders [2-4]. Insomnia in individuals who are alcohol-dependent and actively drinking may aggravate existing psychosocial problems [5]. The prevalence of insomnia in individuals with alcohol dependence is estimated to be between 36 and 91\%. After 2 weeks of alcohol detoxification, as many as $65 \%$ of these individuals still experience sleep problems [2].

\section{Cognitive behavioral therapy for insomnia (CBT-I) as a first-line treatment for insomnia}

CBT-I is an efficacious non-pharmacological treatment for insomnia [6] and is recommended as a first-line treatment for adults with chronic insomnia disorder [7]. It is also effective for those with posttraumatic stress disorder (PTSD) and depression, which are common co-morbidities among those with AUD $[8,9]$. CBT-I has been associated with more rapid and durable improvement in sleep outcomes, even when compared with other non-pharmacologic treatments [10, 11]. While pharmacological intervention for insomnia combined with CBT-I can produce added benefits acutely, medications should ultimately be discontinued for long-term outcomes during "maintenance" CBT-I in order to reduce the chance of insomnia remission [12].

\section{Efficacy of Internet-delivered CBT-I}

Internet-based CBT-I could play a key role in the dissemination of behavioral sleep interventions, given the paucity of trained clinicians able to provide CBT-I in-person and other logistical/cost concerns. This method of delivery is of particular interest to researchers and clinicians working with individuals with AUD [13]. A recent systematic review and meta-analysis of 11 randomized controlled trials (total of 1460 participants) revealed that online CBT-I improved insomnia severity, sleep efficiency, subjective sleep quality, wake after sleep onset, sleep onset latency, total sleep time, and number of nocturnal awakenings with effect sizes comparable to face-to-face CBT-I [14]. Another meta-analysis showed that Internet-based CBT-I significantly improved comorbid anxiety and depression [15]. The most recent meta-analysis of 15 studies showed improved sleep efficiency and total sleep time, while decreasing insomnia severity and depressive symptoms that was maintained from 4 to 48 weeks after the post-treatment assessment [16].

\section{Description of Sleep Health Using the Internet (SHUTi) program}

The SHUTi program is an automated, interactive, Internet-based intervention based on well-established face-to-face CBT-I components including sleep restriction, stimulus control, sleep hygiene, cognitive restructuring, and relapse prevention. It consists of six core areas of focus (Fig. 1) that include interactive educational content and case studies about insomnia and its precipitating factors. These Cores are designed to parallel traditional (in-person) CBT-I sessions and are "metered" out over time; that is, new Cores are available seven days after completion of the previous core. The program includes a variety of interactive features including goal-setting, feedback based on user-entered data and user-identified symptoms, animations, quizzes, vignettes, and video-based expert explanations. SHUTi was developed based on the Model for Internet Interventions $[17,41]$. Sleep diary input is used to customize the program to individual situations. In adults with primary insomnia, SHUTi significantly improved the Insomnia Severity Index (ISI) scores and these improvements were sustained at a 6-month follow-up. Additionally, there were significant decreases in wake after sleep onset (WASO) and increases in sleep efficiency compared to a wait-list control group [17, 41], with similar effects in adult cancer survivors [18]. Evaluation of co-occurring symptoms in those trials showed that SHUTi also significantly improved psychological symptoms, mental health-related quality of life, and fatigue [19]. More recently, researchers established long-term (1 year follow-up) effectiveness in a representative sample with chronic insomnia and a range of comorbid conditions via RCT [20]. Finally, the program has significantly lowered depressive symptoms among those with both insomnia and depression symptoms (without major depressive disorder) compared to timeand attention-matched controls [22].

\section{Gap in the literature/purpose of study}

Despite the established efficacy of CBT-I in the community and in those with AUD, very little is known about the efficacy of Internet-based CBT-I among individuals with AUD [13]. In phase I of the trial described herein, we will assess the feasibility and acceptability of Internet-based CBT-I among treatment-seeking individuals with AUD in recovery. In phase II, we will compare the efficacy of CBT-I versus an education-only control group with respect to primary and secondary outcome variables. Primary outcome variables include changes in 


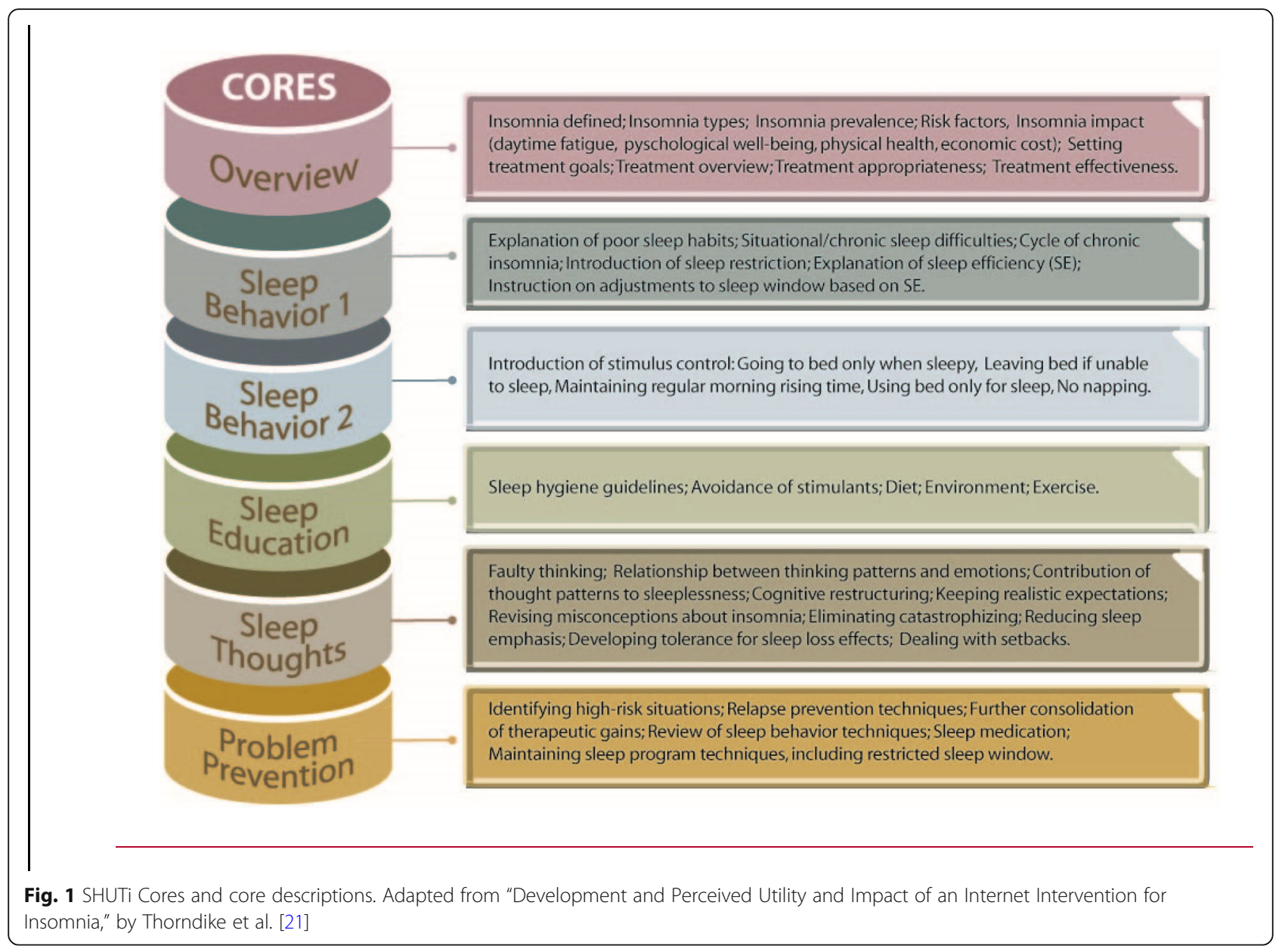

both self-reported insomnia severity and actigraphy-recorded sleep efficiency over time. Secondary outcome variables include additional actigraphy-related outcomes, alcohol craving and consumption, sleep disturbance, daytime sleepiness, anxiety/depression, fatigue, self-efficacy for sleep, dysfunctional beliefs about sleep, and functional outcomes.

\section{Study design and methods}

All participants enrolled in this study will have first been admitted to a clinical research facility providing rehabilitation treatment (NIH Clinical Center) under a screening and assessment protocol, which enrolls adults over 18 years of age seeking inpatient treatment for AUD. Once consented and enrolled into inpatient treatment, screening, and research protocol, participants are screened and recruited to participate in additional studies for which they meet eligibility criteria (including the study described herein). In phase I of this study, all participants will be given access to the SHUTi intervention. Phase I will assess the feasibility and acceptability of the intervention and ensure that our data collection processes are functioning as they should be. Participants will receive training from a member of the research team on accessing the program and using the handheld tablets provided as part of study participation.

In phase II, we will randomize participants to each of the two conditions. The study team will remain blind to the randomization scheme until each study participant is deemed eligible and signs the consent form. Permuted block randomization with a block size of four will be used to equally assign participants to each group. The statistician, who will not have any contact with participants, will prepare the randomization sequences and provide the allocation assignment after each participant is consented. Individuals assigned to the intervention group will complete six sessions ("Cores") of SHUTi intervention (at least one Core completed while inpatient, the rest while outpatient). The SHUTi program tailors specific recommendations based on sleep diary responses or other input within the program (e.g., responses on the Dysfunctional Beliefs and Attitude Scale trigger recommendations for specific cognitive restructuring strategies). The SHUTi program has the ability to send automated pre-developed introductory, reminder, congratulatory, and (insomnia) relapse prevention emails 
to participants, which serve to enhance engagement and clinical outcomes. Individuals assigned to the control group will have access to an insomnia education web-based program that participants access and read at their own pace. The program contains information about insomnia symptoms, impacts, and causes, when to see a doctor, and basic strategies to improve sleep. While the content of the educational program overlaps with that of SHUTi, the education-only program contains no interactive features and delivers content all at once (participants need not wait for content to be "unlocked" over time). Additionally, the educational program contains no customization of the program based on sleep diary responses. Instead, participants in the control group are given educational information (like they might see on WebMD or National Sleep Foundation websites), but are left to apply it themselves.

Study participants will be asked to come back to the $\mathrm{NIH}$ Clinical Center post-discharge for a follow-up visit to complete a face-to-face semi-structured interview (phase I participants only) and assessments (phases I and II participants). If participants are unable to return for the follow-up visit, we will attempt to conduct the surveys and interview via phone. Participants who are randomly assigned to the intervention condition will be granted access to the program free of charge for up to 6 months from the start of the program. Participants who are randomly assigned to the education-only condition will be given access to the full SHUTi program 3 months after discharge upon request. Similarly, participants who are randomly assigned to the intervention condition will be granted access to the educational materials provided to the education-only condition participants 3 months after discharge upon request.

\section{Study measures}

\section{Screening measures}

Screening measures will include the Insomnia Severity Index (ISI), which is a seven-item global index of self-reported insomnia symptom severity [23] and an objective measure of each individual's Apnea-Hypopnea Index (AHI) to determine presence/severity of obstructive sleep apnea (OSA) measured with the WatchPAT. The WatchPAT is a portable diagnostic medical device that works by using Peripheral Arterial Tone (PAT) technology that allows for non-invasive and portable detection of sleep apnea [24]. WatchPAT-generated AHI was shown to have a high correlation with polysomnography (PSG)-generated AHI $(r=0.88-0.92)$ making it a good alternative to PSG as an OSA diagnostic tool [2527]. The results of the WatchPAT study will be reviewed with the patient and the clinical care team. If the patient's results suggest mild obstructive sleep apnea (5-14.9 events/hour of sleep), we will recommend him/her to work on losing weight (if they are overweight or obese), avoid alcohol use, and follow up with a primary care provider. In the case that they are found to have moderate to severe OSA (score of 15 or higher), we will provide the patient with a patient education handout that explains what OSA is, the risks associated with untreated OSA, and the treatment options available. We will urge the patient to seek evaluation and treatment through a primary care provider or directly from a sleep medicine clinic, as is appropriate for them.

\section{Feasibility and acceptability measures}

Participants enrolled during phase I will be asked to start the SHUTi Cores prior to being discharged from inpatient treatment. They will be invited to participate in a short interview after they complete the first SHUTi Core in order to gather additional information on program delivery and acceptability. We will also utilize the Internet Evaluation and Utility Questionnaire [28] at the pre-discharge interview, which is a 16-item measure designed to assess usability, likeability, usefulness, understandability, and convenience of the intervention. Open-ended questions (used in phase I only) will be audio recorded and the entire session (including the questionnaires) is expected to last between 20 and $60 \mathrm{~min}$, depending on participants' responses.

We will implement an exit assessment for phase I and II participants. At the exit assessment, we will use the Internet Impact and Effectiveness Questionnaire, the Internet Evaluation and Utility Questionnaire, and the Internet Intervention Adherence Questionnaire [28]. Participants will complete these assessments with a member of the research team who will take notes on participants' comments for each question. Additional measures of program adherence (in the experimental group, for both phases I and II) will be measured by three variables: login count (goal of two per week), completed diary count (minimum of five per week), and number of "Cores" completed out of a possible six Cores.

\section{Outcome measures}

Outcome measures administered at the pre- and post-test assessment (see Additional file 1 for timing of administration) include the Insomnia Severity Index (ISI), daily sleep and symptom diaries, the Penn Alcohol Craving Scale [29], the Functional Outcomes of Sleep Questionnaire (FOSQ-10; [30]), the Pittsburgh Sleep Quality Index (PSQI; [31]), the Multidimensional Fatigue Symptom Inventory-Short Form (MFSI-SF; [32]), the Epworth Sleepiness Scale (ESS; [33]), the Self-Efficacy for Sleep Scale (SE-S; [34]), the Dysfunctional Beliefs and Attitudes about Sleep Scale (brief version; DBAS-16; [35]), the Inventory of Depressive Symptoms (IDS; 30-item self-rated version; [36]), the Trait Anxiety 
Inventory [37], the Composite Scale of Morningness (CSM; [38]), and measures of relapse to include a dichotomous (yes/no) assessment and Timeline Follow-Back (TLFB; [39]).

\section{Objective measure of sleep: actigraphy}

"Actiwatches" are small wristband data loggers which contain accelerometers and light sensors in order to objectively assess sleep. We have used the Actiwatch Spectrum Plus (Philips Respironics) in previous studies with patients undergoing alcohol rehabilitation in both inpatient [40] and outpatient settings. All study participants will be asked to wear an actiwatch for 4 days (capturing three nights' worth of sleep data) at three study time points. The main outcome of interest will be improvements in sleep efficiency from baseline to follow-up assessment periods. The watch also provides measures of total sleep time, wake after sleep onset, sleep onset latency, number of awakenings, and time in bed.

\section{Inclusion and exclusion criteria}

Participants will be eligible for this study if they are at least 18 years of age, self-report "moderate or severe" insomnia as indicated by a score of 15 or higher on the Insomnia Severity Index, are admitted as a treatment-seeking inpatient at the NIH Clinical Center under protocol 14-AA-0181 (signing both the clinical and research consent), have been an inpatient for at least 14 days prior to consent/screening, can speak, understand, and write in English, and are able to comply with study requirements (including ability to access the Internet at least two times per week). Participants will be ineligible for this study if they are over the age of 65 , report a physician diagnosis of moderate to severe obstructive sleep apnea (OSA) or test positive for moderate to severe OSA as documented with an Apnea Hypopnea Index of $\geq 15$ events/hour based on WatchPAT testing results, have irregular sleep schedules that prevent the ability to follow treatment recommendations (i.e., usual bedtimes outside of 8:00 pm to 2:00 am or arising times outside of 4:00 am to 10:00 am), meet criteria for severe opioid and/or cocaine use disorder in the past year, meet criteria for moderate to severe cannabis use disorder in the past year, meet diagnostic criteria for an unstable or serious psychiatric condition (schizophrenia, bipolar, major depressive disorder not currently in remission), based on the SCID for DSM-5, or have an unstable or serious medical/neurologic illness as identified by the Principal Investigator or Medical Advisory Investigator. Participants will be discontinued/withdrawn from the study if any of the following events occur: participant requests to be removed from study, participant is admitted to an addiction inpatient treatment program for 2 weeks or longer, participant exhibits any condition which the Medical Advisory Investigator finds CBT-I to be a hazard to the participant (i.e., participant seems agitated, onset of increasing paranoia), participant is unable to comply with study-related procedures (i.e., SHUTi program recommendations and/or "homework"), and/or participant develops a medical illness or condition that requires a new hospital admission.

\section{Power analysis}

In studies of CBT-I, the effect sizes for improvements in insomnia severity ranged from $d=0.2$ [20] to $d=2.24$ [18], with the sample sizes ranging from 29 [18] to 300 individuals [20]. One such study also provided effect sizes for improvements in several other sleep-related variables, including wake after sleep onset $(d=0.74)$, sleep onset latency $(d=0.26)$, and sleep efficiency $(d=0.68 ;[17,41])$. In phase I, our sample size is based on the concept of saturation, which describes the number of participants at which no new (qualitative) key themes are likely to be generated. Based on our previous qualitative research in the same population, it is expected that 12 patients will be screened to achieve 10 "completers" (i.e., individuals providing pre- and post-test assessment data). The total sample size for phase II of this study will test the primary hypotheses associated with the analysis plan. With insomnia severity as the primary outcome, we will use a two-sided alternative hypothesis that the rate of change will be different between the intervention group and the control group. Assuming a correlation of 0.6 between the two time points, an alpha level of 0.05 , a power level of 0.8 , and an estimated attrition rate of $40 \%$, we will be able to detect an effect size of 0.84 with 60 participants [42].

\section{Justification for specific inclusion/exclusion criteria}

Clinical insomnia is considered to be moderate to severe insomnia and is the target of treatment (refer to Bastien et al. 2001 [23], Fig. 1 for reference). In order to demonstrate efficacy, treatment in patients with "clinical" insomnia is considered appropriate and will help obtain estimates of effect sizes. Sub-clinical insomnia may remit on its own without treatment, thus making it unclear whether the intervention improved insomnia or it was a case of spontaneous remission of insomnia. One of CBT-I's potential side effects from its sleep restriction phase is increased daytime sleepiness. Increased daytime sleepiness is associated with increased frequency of falls in the elderly (individuals over 65 years old; [43]); thus, we are excluding individuals over the age of 65 . Among individuals who are alcohol-dependent with untreated moderate-severe OSA, sleep restriction may further increase sympathetic and stress response, leading to an increased risk of cardiovascular adverse events [44]. Severe OSA is associated with increased daytime sleepiness, motor vehicle crashes, depression, cardiovascular and cerebral morbidity and mortality, and cognitive and 
metabolic dysfunction [45]. Thus, we are excluding individuals with untreated moderate to severe OSA. A study by Pacek et al. [46] reported that participants reported sleep difficulties with abstinence from cannabis and resulted in the relapse in cannabis use at a later time. It is unclear whether sleep difficulty symptoms improve with relapse, but the authors also point out that cannabis use contributes to observed sleep difficulties. Whether or not disordered sleep contributes to long-term cannabis use is unclear [46]. Finally, cannabis is independently associated with impairment of sleep continuity [46]. Opioids are associated with a more complex picture of sleep disorders that includes insomnia, obstructive sleep apnea, and complex sleep apnea [47]. Thus, we are excluding participants with severe opioid/cocaine use disorder in the past year and/or moderate-severe cannabis use disorder in the past year. After enrollment in the study, the management of sedatives/hypnotics for individuals who have been prescribed either will be done by a clinician who is blind to the treatment condition.

\section{Recruitment plan}

All eligible participants admitted to the NIH Clinical Center alcohol rehabilitation unit who signed the clinical and research consents for the screening/treatment protocol will be approached for participation in this study. The Principal Investigator or a trained Associate Investigator approved by the IRB will explain the study objectives, time commitment, expectations, and processes for assessments.

\section{Statistical analysis}

\section{Qualitative analysis (phase I only)}

Each audio-recorded interview will be transcribed verbatim. First, independent coders will review all transcripts and identify common themes. Once the theme list is agreed on, after thematic analysis, a codebook will be developed based on themes from the interviews. Each code will be accompanied by an operational definition that will allow for clarity and consistency in the coding process. Evidence of each code or theme will be assessed using quotes from the interviews. A team of coders will independently review all transcripts. Discordant coding will be discussed until consensus among the coding team is achieved. Once the iterative process of consensus building is complete, an intramural expert in qualitative methodology will validate the final themes and coding. If this final reviewer notes any discrepancies during this validation process or if they identify an additional theme not previously noted, the coded themes will be returned to the team of coders for further consensus. After data are coded, NVivo will be utilized for further qualitative analysis and data management.

\section{Quantitative analyses (phase II only)}

Patterns of missing data will be examined thoroughly to assess whether any questions were systematically skipped by all participants or any sub-group of participants. Initial analyses will be descriptive and exploratory in nature to identify any changes in insomnia severity (as measured by the ISI) over time. Mixed models for repeated measure with group, time, and the interaction of group by time will be used to test the changes of insomnia severity in two groups. Mixed models for repeated measures will also be used to test the secondary objectives with potential covariates in the model. Demographic, psychiatric, and/or drinking-related factors significantly related to the outcomes listed in the secondary objectives will be entered in the model. Akaike information criterion and the Bayesian information criterion will be used to select best-fitting models. A potential dosing effect analysis including the number of Cores completed as a covariate will be conducted for the treatment group only. With the linear mixed model approach, all available data points will be included in the analyses. Under missing completely at random and missing at random assumptions, a linear mixed model with restricted maximum likelihood estimation can produce unbiased estimators [48].

\section{Actigraphy analyses}

After device removal and data download, raw data from the Actiwatch Spectrums will be analyzed using the Philips Respironics computerized sleep scoring software, which scores each epoch based on a threshold method algorithm. Investigators will review each sleep period prior to analysis to screen for malfunctioning watches, corrupt data, and required adjustments using bedtimes and wake times from self-reported diary data when necessary.

\section{Evaluation of risks/discomforts and benefits ratio}

This study will provide pilot data on the feasibility, acceptability, and preliminary efficacy of an Internet-based CBT-I program undergoing inpatient treatment AUD. The program may represent an efficacious non-pharmacologic intervention for insomnia in this population, potentially decreasing the need and use of a pharmacological intervention. However, it is possible that there may be no direct benefit to the participant. It is possible that answering some of the questionnaires may cause discomfort to participants. Participants may feel uncomfortable wearing the WatchPAT device or the actiwatch device to sleep. Participants may experience a rash and/or pruritus while wearing the WatchPAT or actiwatch. Participants in phase I and those assigned to the SHUTi condition in phase II may experience daytime sleepiness (and potentially impaired daytime functioning), particularly if they are taking medications with side effects of drowsiness during the study period. Every effort will be made to address and 
minimize participant discomfort including scheduling a repeat session or postponing study procedures if a participant becomes uncomfortable. Confidentiality and information technology security standards are in place as part of the $\mathrm{NIH}$ Clinical Center intramural program to protect electronic repositories of patient data. We selected a minimally invasive diagnostic tool to assess for OSA, requiring only one night of sleeping with the device. It is reasonably expected that these safeguards will protect participants' medical and personal health information, ensuring their privacy.

\section{Data/records management}

The PI will be responsible for assuring that all investigators follow the plan for protecting the confidentiality of information and data provided by research participants. Information to be collected via the Internet-based insomnia treatment program, SHUTi, will include participants' responses to individual questions within the program and the dates and times questions and Cores are completed. Participants will not be asked to provide any personally identifiable information in the course of their engagement in the SHUTi intervention and/or education-only programs. At the initial meeting for consenting and baseline assessment, participants will be provided with a unique user login ID and a temporary password. The participant will be required to change the password at initial logon to the website to a personal one that he or she will recall. Only the participant and the study staff will have the authority to access the participant's responses to the components of the intervention. All baseline and follow-up assessments not part of the SHUTi program will be completed in person or over the phone with paper-and-pencil questionnaires and will be administered by a member of the research team.

\section{Compensation}

Participants will be compensated for research-related discomfort and inconveniences in accordance with $\mathrm{NIH}$ guidelines. If participants are unable to finish the study, they will be paid only for those parts completed. Because participants will need access to a tablet or laptop to complete the program, we will provide tablets to all participants (capable of Wifi connectivity). Participants will be able to keep the study-provided tablets.

\section{Strengths and limitations}

This study is not without possible limitations. Participants must be comfortable using a tablet; thus, study team members will provide informal training. Also, all participants will be recruited from a clinical research facility providing rehabilitation and must be able to comply with study requirements (i.e., using a tablet) so it may be difficult to generalize results. For instance, participants spend an average of about 31 days in inpatient treatment, and sleep hygiene on the inpatient unit may be more regulated compared to at home. Cross-contamination of study arms may occur, particularly since participants will be in close proximity in an inpatient setting. Participants will be encouraged to not share information about SHUTi or the education component with other patients on the unit. The strengths of this study lie in the use of both subjective and objective (actigraphy) assessments of sleep, validated patient-reported outcome measures, and high potential for dissemination in real-world settings. Examining potential correlates of intervention outcome will allow us to identify further sub-groups of individuals to target for interventions in the future.

\section{Conclusions}

This study addresses a critical gap in our understanding of the utility of Internet-based CBT-I in a vulnerable population. While insomnia and other sleep disturbances are prevalent among individuals with AUD, to date, no randomized controlled trials have been conducted to establish the feasibility, acceptability, and preliminary efficacy of an Internet-based CBT-I program among individuals with comorbid insomnia and AUD. Understanding the utility of SHUTi and other behavioral interventions like it could help clinicians who support individuals with AUD in recovery identify useful, accessible, and efficacious treatments for use in this population. From a public health perspective, a low-cost, low-risk behavioral intervention for insomnia such as SHUTi could be implemented widely in both clinical and community settings.

\section{Trial status}

This study was approved by the NIH Addictions Institutional Review Board in March of 2018 (NCT\#03493958). We expect to begin recruitment in June of 2018.

\section{Additional file}

Additional file 1: Study Schema and Timing of Assessments. Timing of assessment of outcome measures. (DOCX $19 \mathrm{~kb}$ )

\section{Abbreviations}

AUD: Alcohol use disorder; CBT-I: Cognitive behavioral therapy for insomnia; CSM: Composite Scale of Morningness; DSM: Diagnostic and Statistical Manual; ESS: Epworth Sleepiness Scale; FOSQ: Functional Outcomes of Sleep Questionnaire; ISI: Insomnia Severity Index; MFSI-SF: Multidimensional Fatigue Symptom Inventory-Short Form; NIH: National Institutes of Health;

PACS: Penn Alcohol Craving Scale; PSQI: Pittsburgh Sleep Quality Index; SE-S: Self-Efficacy for Sleep Scale; SHUTi: Sleep Healthy Using the Internet

\section{Acknowledgments}

Not applicable.

Funding

This project is funded with federal funds from the National Institutes of Health, Clinical Center intramural research program. The content of this publication does not necessarily reflect the views or policies of the Department of Health 
and Human Services, nor does mention of trade names, commercial products, or organizations imply endorsement by the U.S. Government.

\section{Availability of data and materials}

Data sharing is not applicable to this article as no datasets were generated or analyzed during the current study.

\section{Authors' contributions}

ATB, RTT, LMR, FPT, SR, and GRW contributed to the study design and preparation of the manuscript. SC, LMR, and FPT consulted on study assessments. All authors read and approved the final manuscript.

\section{Ethics approval and consent to participate}

This study was approved by the NIH Addictions Institutional Review Board in March of 2018. All study participants will provide written informed consent.

\section{Consent for publication}

Not applicable.

\section{Competing interests}

Drs. Ritterband and Thorndike have equity ownership in BeHealth Solutions, LLC, a company developing and making available products related to the research reported in this publication. Specifically, BeHealth Solutions, LLC has licensed the SHUTi program and the software platform on which it was built from the University of Virginia. The terms of this arrangement have been reviewed and approved by the University of Virginia and the National Institutes of Health in accordance with their conflict of interest policies.

\section{Publisher's Note}

Springer Nature remains neutral with regard to jurisdictional claims in published maps and institutional affiliations.

\section{Author details}

${ }^{1}$ National Institutes of Health Clinical Center, Bethesda, USA. ${ }^{2}$ Perelman School of Medicine, Department of Psychiatry, University of Pennsylvania, Philadelphia, USA. ${ }^{3}$ Department of Psychiatry and Neurobehavioral Sciences, Center for Behavioral Health and Technology, PO Box 801075, Charlottesville, VA 22908, USA. ${ }^{4}$ Pear Therapeutics, Boston, USA.

\section{Received: 9 July 2018 Accepted: 25 November 2018} Published online: 10 December 2018

\section{References}

1. Alcohol Use Disorder. National Institute on Alcohol Abuse and Alcoholism (NIAAA). 2017. https://www.niaaa.nih.gov/alcohol-health/overview-alcoholconsumption/alcohol-use-disorders. Accessed 10 Mar 2017.

2. Chakravorty S, Chaudhary NS, Brower KJ. Alcohol dependence and its relationship with insomnia and other sleep disorders. Alcohol Clin Exp Res. 2016. https://doi.org/10.1111/acer.13217.

3. Stein MD, Friedmann PD. Disturbed sleep and its relationship to alcohol use. Subst Abus. 2005;26:1-13.

4. Brower KJ. Alcohols' effect on sleep in alcoholics. Alcohol Res Health. 2001 25:110-25.

5. Chaudhary NS, Kampman KM, Kranzler HR, Grandner MA, Debbarma S, Chakravorty S. Insomnia in alcohol dependent subjects is associated with greater psychosocial problem severity. Addict Behav. 2015. https://doi.org/ 10.1016/j.addbeh.2015.06.021

6. Morin CM, Bootzin RR, Buysse DJ, Edinger JD, Espie CA, Lichstein KL. Psychological and behavioral treatment of insomnia:update of the recent evidence (1998-2004). Sleep. 2006;29:1398-414.

7. Qaseem A, Kansagara D, Forciea MA, Cooke M, Denberg TD. Management of chronic insomnia disorder in adults: a clinical practice guideline from the American College of Physicians. Ann Intern Med. 2016. https://doi.org/10. 7326/m15-2175.

8. Talbot LS, Maguen S, Metzler TJ, Schmitz M, McCaslin SE, Richards A, et al. Cognitive behavioral therapy for insomnia in posttraumatic stress disorder: a randomized controlled trial. Sleep. 2014. https://doi.org/10.5665/sleep.3408.

9. Siebern AT, Manber R. New developments in cognitive behavioral therapy as the first-line treatment of insomnia. Psychol Res Behav Manag. 2011. https://doi.org/10.2147/prbm.s10041.
10. Garland SN, Carlson LE, Stephens AJ, Antle MC, Samuels C, Campbell TS Mindfulness-based stress reduction compared with cognitive behavioral therapy for the treatment of insomnia comorbid with cancer: a randomized, partially blinded, noninferiority trial. J Clin Oncol. 2014. https://doi.org/10. 1200/jco.2012.47.7265.

11. Miller KE, Kuhn E, Owen JE, Taylor K, Yu JS, Weiss BJ, et al. Clinician perceptions related to the use of the CBT-I coach mobile app. Behav Sleep Med. 2017. https://doi.org/10.1080/15402002.2017.1403326.

12. Morin CM, Vallières A, Guay B, Ivers H, Savard J, Merette $C$, et al. Cognitive behavioral therapy, singly and combined with medication, for persistent insomnia: a randomized controlled trial. JAMA. 2009. https://doi.org/10. 1001/jama.2009.682.

13. Brooks AT, Wallen GR. Sleep disturbances in individuals with alcohol-related disorders: a review of cognitive-behavioral therapy for insomnia (CBT-I) and associated non-pharmacological therapies. Subst Abus. 2014. https://doi. org/10.4137/sart.s18446.

14. Zachariae R, Lyby MS, Ritterband LM, OToole MS. Efficacy of internetdelivered cognitive-behavioral therapy for insomnia - a systematic review and meta-analysis of randomized controlled trials. Sleep Med Rev. 2016. https://doi.org/10.1016/j.smrv.2015.10.004.

15. Ye YY, Zhang YF, Chen J, Liu J, Li XJ, Liu YZ, et al. Internet-based cognitive behavioral therapy for insomnia (ICBT-i) improves comorbid anxiety and depression-a meta-analysis of randomized controlled trials. PLoS One. 2015. https://doi.org/10.1371/journal.pone.0142258.

16. Seyffert M, Lagisetty P, Landgraf J, Chopra V, Pfeiffer PN, Conte ML, Rogers MA. Internet-delivered cognitive behavioral therapy to treat insomnia: a systematic review and meta-analysis. PLoS One. 2016. https://doi.org/10 1371/journal.pone.0149139.

17. Ritterband LM, Thorndike FP, Gonder-Frederick LA, Magee JC, Bailey ET, Saylor DK, Morin CM. Efficacy of an internet-based behavioral intervention for adults with insomnia. Arch Gen Psychiatry. 2009. https://doi.org/10.1001/ archgenpsychiatry.2009.66.

18. Ritterband LM, Bailey ET, Thorndike FP, Lord HR, Farrell-Carnahan L, Baum LD. Initial evaluation of an internet intervention to improve the sleep of cancer survivors with insomnia. Psychooncology. 2012. https://doi.org/10. 1002/pon.1969.

19. Thorndike FP, Ritterband LM, Gonder-Frederick LA, Lord HR, Ingersoll KS, Morin CM. A randomized controlled trial of an internet intervention for adults with insomnia: effects on comorbid psychological and fatigue symptoms. J Clin Psychol. 2013. https://doi.org/10.1002/jclp.22032.

20. Ritterband LM, Thorndike FP, Ingersoll KS, Lord HR, Gonder-Frederick L, Frederick $C$, et al. Effect of a web-based cognitive behavior therapy for insomnia intervention with 1-year follow-up: a randomized clinical trial. JAMA Psychiatry. 2017. https://doi.org/10.1001/jamapsychiatry.2016.3249.

21. Thorndike FP, Saylor DK, Bailey ET, Gonder-Frederick L, Morin CM, Ritterband LM. Development and perceived utility and impact of an Internet intervention for insomnia. E J Appl Psychol. 2008;4(2):32-42.

22. Christensen H, Batterham PJ, Gosling JA, Ritterband LM, Griffiths KM, Thorndike FP, et al. Effectiveness of an online insomnia program (SHUTi) for prevention of depressive episodes (the GoodNight study): a randomised controlled trial. Lancet Psychiatry. 2016. https://doi.org/10.1016/s2215-0366(15)00536-2.

23. Bastien $\mathrm{CH}$, Vallieres $\mathrm{A}$, Morin CM. Validation of the insomnia severity index as an outcome measure for insomnia research. Sleep Med. 2001;2:297-307.

24. PAT (Peripheral Arterial Tone) Technology A New Signal for Health Being. Itamar Medical. 2016. http://www.itamar-medical.com/pat-technologywatchpat/. Accessed 18 May 2018.

25. Tanphaichitr A, Thianboonsong A, Banhiran W, Vathanophas V, Ungkanont K. Watch peripheral arterial tonometry in the diagnosis of pediatric obstructive sleep apnea. Otolaryngol Head Neck Surg. 2018. https://doi.org/ 10.1177/0194599818768215.

26. Weimin L, Rongguang W, Dongyan H, Xiaoli L, Wei J, Shiming Y. Assessment of a portable monitoring device WatchPAT 200 in the diagnosis of obstructive sleep apnea. Eur Arch Otorhinolaryngol. 2013. https://doi.org/ 10.1007/s00405-013-2555-4.

27. Yalamanchali S, Farajian V, Hamilton C, Pott TR, Samuelson CG, Friedman M. Diagnosis of obstructive sleep apnea by peripheral arterial tonometry: metaanalysis. JAMA Otolaryngol Head Neck Surg. 2013. https://doi.org/10.1001/ jamaoto.2013.5338.

28. Ritterband LM, Ardalan K, Thorndike FP, Magee JC, Saylor DK, Cox DJ, et al. Real world use of an internet intervention for pediatric encopresis. J Med Internet Res. 2008. https://doi.org/10.2196/jmir.1081. 
29. Flannery BA, Volpicelli JR, Pettinati HM. Psychometric properties of the Penn alcohol craving scale Alcohol Clin Exp Res. 1999;23:1289-95.

30. Chasens ER, Ratcliffe SJ, Weaver TE. Development of the FOSQ-10: a short version of the Functional Outcomes of Sleep Questionnaire. Sleep. 2009;32: 915-9.

31. Buysse DJ, Reynolds CF 3rd, Monk TH, Berman SR, Kupfer DJ. The Pittsburgh Sleep Quality Index: a new instrument for psychiatric practice and research. Psychiatry Res. 1989;28:193-213.

32. Stein $\mathrm{KD}$, Jacobsen PB, Blanchard CM, Thors C. Further validation of the multidimensional fatigue symptom inventory-short form. J Pain Symptom Manag. 2004;27:14-23.

33. Johns, MW. A new method for measuring daytime sleepiness: the Epworth sleepiness scale. Sleep. 1991.

34. Edinger JD, Wohlgemuth WK, Radtke RA, Marsh GR, Quillian RE. Cognitive behavioral therapy for treatment of chronic primary insomnia: a randomized controlled trial. JAMA. 2001;285:1856-64.

35. Morin CM, Vallieres A, Ivers H. Dysfunctional beliefs and attitudes about sleep (DBAS): validation of a brief version (DBAS-16). Sleep. 2007;30:1547-54.

36. Rush AJ, Trivedi MH, Ibrahim HM, Carmody TJ, Arnow B, Klein DN, et al. The 16-item Quick Inventory of Depressive Symptomatology (QIDS), clinician rating (QIDS-C), and self-report (QIDS-SR): a psychometric evaluation in patients with chronic major depression. Biol Psychiatry. 2003. https://doi. org/10.1016/50006-3223(02)01866-8.

37. Spielberger CD, Gorsuch RL, Lushene R, Vagg PR, Jacobs GA. Manual for the state-trait anxiety inventory STAI (Form Y). Palo Alto: Consulting Psychologists Press; 1983.

38. Smith CS, Reilly C, Midkiff K. Evaluation of three circadian rhythm questionnaires with suggestions for an improved measure of morningness. J Appl Psychol. 1989;74:728-38.

39. Sobell LC, Sobell MB. Timeline follow-back: a technique for assessing selfreported ethanol consumption. In: Allen J, Litten R, editors. Measuring alcohol consumption: psychosocial and biochemical methods. Totowa: Humana Press; 1992. p. 42-72.

40. Wallen GR, Brooks AT, Whiting B, Clark R, Krumlauf MC, Yang L, et al. The prevalence of sleep disturbance in alcoholics admitted for treatment: a target for chronic disease management. Fam Community Health. 2014. https://doi.org/10.1097/FCH.0000000000000040.

41. Ritterband LM, Thorndike FP, Cox DJ, Kovatchev BP, Gonder-Frederick LA. A behavior change model for internet interventions. Ann Behav Med. 2009. https://doi.org/10.1007/s12160-009-9133-4.

42. Fitzmaurice GM, Laird NM, Ware JH. Applied longitudinal analysis. Vol. 998. Hoboken: Wiley; 2012.

43. Helbig AK, Doring A, Heier M, Emeny RT, Zimmermann AK, Autenrieth CS, et al. Association between sleep disturbances and falls among the elderly: results from the German Cooperative Health Research in the Region of Augsburg-Age study. Sleep Med. 2013. https://doi.org/10.1016/.sleep.2013.09.004

44. Irwin MR, Ziegler M. Sleep deprivation potentiates activation of cardiovascular and catecholamine responses in abstinent alcoholics. Hypertension. 2005. https://doi.org/10.1161/01.HYP.0000153517.44295.07.

45. Peppard PE, Hagen EW. The last 25 years of obstructive sleep apnea epidemiology-and the next 25? Am J Respir Crit Care Med. 2018. https://doi. org/10.1164/rccm.201708-1614PP.

46. Pacek LR, Herrmann ES, Smith MT, Vandrey R. Sleep continuity, architecture and quality among treatment-seeking cannabis users: an in-home, unattended polysomnographic study. Exp Clin Psychopharmacol. 2017. https://doi.org/10.1037/pha0000126.

47. Hassamal S, Miotto K, Wang TS, Saxon AJ. A narrative review: the effects of opioids on sleep disordered breathing in chronic pain patients and methadone maintained patients. Am J Addict. 2016. https://doi.org/10.1111/ ajad.12424.

48. Salim A, Mackinnon A, Christensen H, Griffiths K. Comparison of data analysis strategies for intent-to-treat analysis in pre-test-post-test designs with substantial dropout rates. Psychiatry Res. 2008. https://doi.org/10.1016/ j.psychres.2007.08.005.

Ready to submit your research? Choose BMC and benefit from:

- fast, convenient online submission

- thorough peer review by experienced researchers in your field

- rapid publication on acceptance

- support for research data, including large and complex data types

- gold Open Access which fosters wider collaboration and increased citations

- maximum visibility for your research: over $100 \mathrm{M}$ website views per year

At BMC, research is always in progress.

Learn more biomedcentral.com/submissions 